\title{
MEDICAL EDUCATION
}

\section{The Relationship of Difficulty and Discrimination Indices with Physiology Teaching and Learning}

\author{
Sadaf Fatima', Syed Tousif Ahmed', Shazia Hashmat', Afshan Iftikhar ${ }^{3}$, Touqeer Alam Hashmi ${ }^{4}$ \\ 'Department of Physiology, Jinnah Medical and Dental College, '2Department of Physiology, Ziauddin Medical College, \\ Ziauddin University, ${ }^{3}$ Department of Physiology, Lahore Medical and Dental college, Lahore, ${ }^{4}$ Department of \\ Examination, Ziauddin University, Karachi, Pakistan.
}

\begin{abstract}
Background: Item analysis, a valuable process conducted after the examination, gives information regarding the reliability of scores and validity of items tested. Difficulty index (P) and Discrimination index (DI) are two parameters, which aid in analyzing the standard of Multiple Choice Questions (MCQs). The study was aimed to find the relationship of difficulty and discrimination indices with teaching and learning in Physiology.
\end{abstract}

Methods: Students $(n=100)$ included were MBBS $1^{\text {st }}$ year from September 2017 to March 2018. The MCQs of Physiology semester II courses were analyzed in two Continuous Assessment Tests (CATs). CAT I included Respiratory: 15/50 and CAT II Hemopoietic: 14/60 and Semester Exam 40/80. The items were examined for difficulty index ( $p$-value) and discrimination index (DI). Items having $p$-value between 0.3 to 0.7 and $\mathrm{DI}>0.2$ were considered as having good difficulty and discrimination indices respectively.

Results: Out of 15 items in Respiratory CAT, $73 \%$ had a mean $p=0.3-0.7$ and $27 \%$ had mean $p=0.23$. The mean DI value was 0.31 . Out of 14 items in CAT of Hemopoietic, $85 \%$ had a $p$-value between $0.3-0.7$ and $15 \%$ had mean $p=0.2$. The mean DI value was 0.41 . Out of 40 items in Semester exam, $82 \%$ items had $p$ value b/w 0.3 -0.7 and $18 \%$ items had mean $p=0.18$. Overall mean DI value was 0.28 .

Conclusion: The item analysis of majority of one best type MCQs of CATs and semester exam in Physiology had good difficulty and discrimination indices and matched teaching and learning strategy in Physiology.

Keywords: Difficulty Index; Discrimination Index; Physiology; Teaching; Learning.

\section{Corresponding Author:}

Dr. Sadaf Fatima

Department of Physiology,

Jinnah Medical and Dental College,

Karachi, Pakistan.

Email: doctorsadafnaqvi@yahoo.com

https://doi.org/10.36283/PJMD10-2/019

\section{INTRODUCTION}

Assessment drives learning because it includes both formative and summative knowledge, and has a strong influence on learning ${ }^{1-2}$. It is considered a vital factor in leading the learners for accomplishing the goal $^{3}$. The assessment of knowledge, skills and competence of undergraduate medical students has great significance because of the demand to be a safe practitioner in future ${ }^{3}$. Physiology is one of the basic science subjects taught to undergradu- ate medical students ${ }^{4}$. It is essential due to its application in clinical practice ${ }^{5}$. In medical education, knowledge of Physiology is assessed routinely in the pre-clinical years ${ }^{6}$.

It is tremendously important that assessment should be valid and adequately discriminate between satisfactory and unsatisfactory performers ${ }^{3}$. One best (OB) Multiple choice question (MCQ) is a frequently used tool to assess the cognitive competence of the student ${ }^{6,7}$. In this type of 
assessment, the student selects the best possible answer from the given list ${ }^{8} . M C Q s$ are widely used because of objectivity ${ }^{3,7}$, removal of assessor's bias ${ }^{3}$ and thorough coverage of the subject matter in a short time $e^{3,7}$. MCQs if formulated correctly, can test higher levels of cognition and differentiate between high and low achievers?

Item analysis analyzes the student responses to individual test items (MCQs) to evaluate the distinctive quality of those items and test as a whole ${ }^{10,11}$. It is a valuable process, which is carried out after examination that gives information regarding the reliability and validity of a test ${ }^{8}$. It allows observing the characteristics of a specific item and can be used to make sure that items are of an acceptable standard to be included in a test, needs to be reviewed or discarded from the test ${ }^{12,13}$.

Difficulty index (P) and Discrimination index (DI) are two parameters that help in examining the standard of $M C Q$ questions used in an examination ${ }^{12}$, with abnormal values signifying poor quality. Difficulty index indicates the percentage of correct answers to total responses; also referred to as the $p$-value ${ }^{14}$. Items having a $p$-value between 0.3-0.7 are regarded as having a good difficulty index as it shows that items are of moderate difficulty ${ }^{7}$. In general, items of moderate difficulty are to be chosen to those that are much easier or much harder ${ }^{14}$. The greater the number of questions in a paper with moderate difficulty, the better is the quality of assessment. The discrimination index (DI), which is also known as the point biserial correlation, reflects discrimination between students with different levels of achievement ${ }^{12}$. For an item to discriminate effectively between weaker and stronger candidates, the stronger candidates should perform better on the item than the weaker candidates $^{12}$. Items having DI $>0.2$ were regarded as having good discrimination index ${ }^{7}$. Such items contribute to the examination's overall ability to discriminate stronger from weaker candidates.

Items writing errors are the technical flaws that if present in any $M C Q$, they can influence student's performance ${ }^{3}$. The MCQs are labeled as poorly developed because of item writing flaws ${ }^{7}$. They include ambiguous stems, negatively worded options, options that are not plausible, contains irrelevant information ${ }^{7}$. All of them affect the quality of the MCQs and makes the assessment process less reliable and valid ${ }^{7}$. It is extremely important to align teaching with assessment. The student performance in exam reflects their learning and understanding of the subject. It is not only important to teach well but it is also essential to design assessment appropriately matched with the teaching. The greater the number of questions with appropriate $p$ value and $\mathrm{Dl}$, the more significant is the assessment. To emphasize the significance of item analysis and to express its association with teaching and learning, this study aimed to find out the relationship of difficulty and discrimination indices with teaching and learning in physiology.

\section{METHODS}

The study design was cross sectional. The study duration was six months from September 2017 to March 2018. In this study, the secondary data was acquired from the examination department in the form of item analysis. The study population included 100 First Year MBBS students. The paper is comprised of one-best MCQs, having 5 options each. The same faculty members who taught the particular topic developed the Physiology MCQs. The physiology MCQs included in CATs of courses taught in semester II, Respiratory system and Hemopoietic system and the Physiology MCQs of the semester II exam, were analyzed. There were $15 / 50$ physiology one-best MCQs in Respiratory system CAT (Figure 1a), 14/60 in Hemopoietic system CAT (Figure 1b) and 40/80 one-best MCQs in the semester exam (Figure 1c). The MCQs were analyzed for difficulty index (p-value) and discrimination index (DI). Items having $p$-value between 0.3-0.7 ${ }^{7}$ and $\mathrm{DI}>0.2^{7}$ were regarded as having good difficulty and discrimination indices respectively.

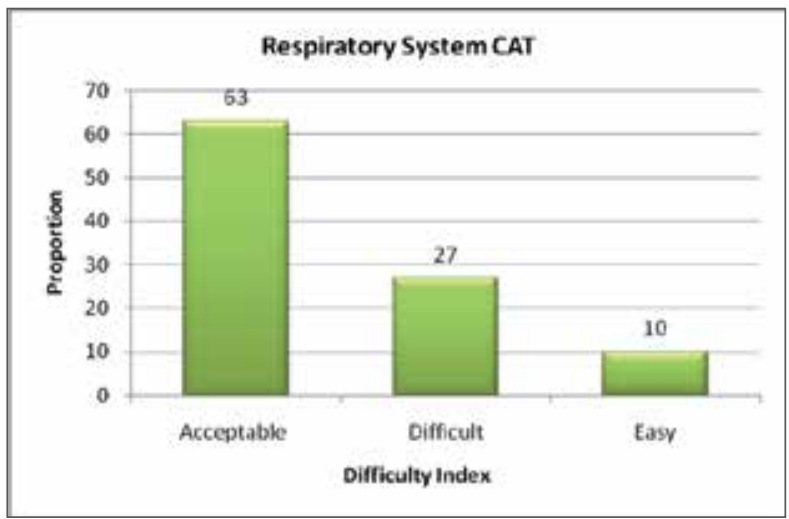

Figure 1a: Proportion of difficulty index in physiology section of respiratory system CAT.

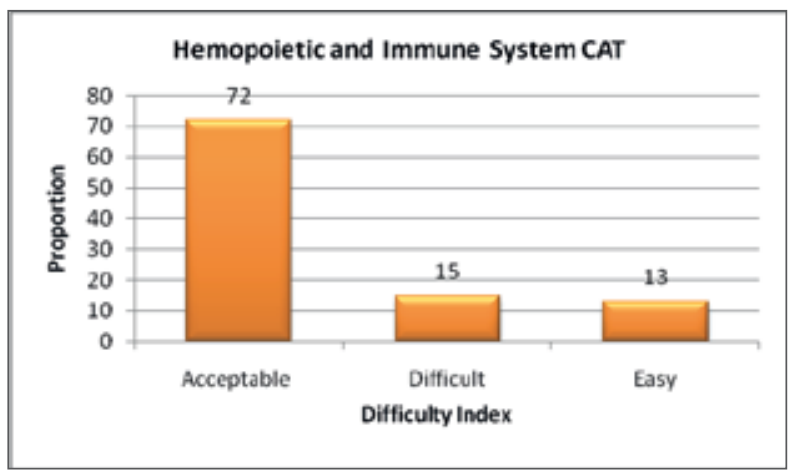

Figure 1b: Proportion of difficulty index in physiology section of hemopoietic and immune system CAT. 
Figure 1c: Proportion of difficulty index in physiology section of semester II Exam.

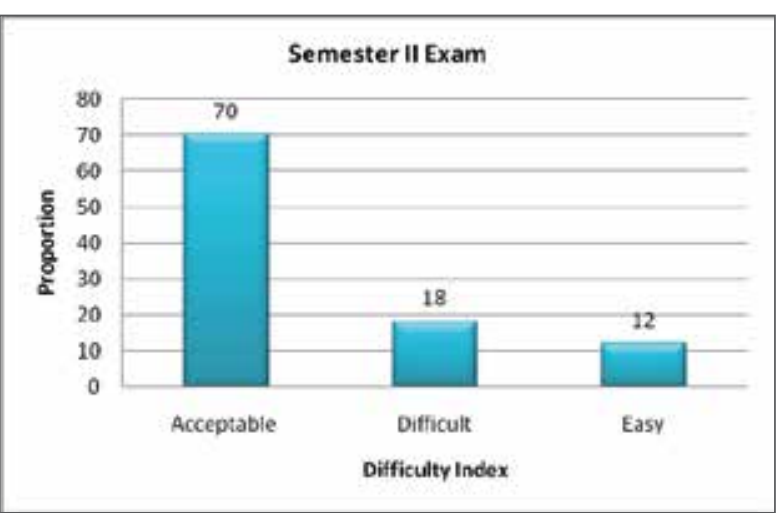

Descriptive statistics were used for analysis of data. Data is expressed in terms of $p$-value and DI. The difficulty index was calculated using the formula $p=R / T$ where $p$ is the item difficulty index, $R$ is the number of correct responses, and $T$ is the total number of responses. The discrimination index was calculated for each item using the formula $\mathrm{DI}=\mathrm{UG}-\mathrm{LG} / \mathrm{n}$ where $\mathrm{DI}$ is discrimination index, UG is a number of students in the upper group who got an item correct, LG is number of students in lower group. The mean $p$-value and mean DI value was calculated.

\section{RESULTS}

Out of 15 Physiology items in CAT of Respiratory system, $73 \%$ were found to have $p$-value b/w 0.3 -0.7 and $27 \%$ items had mean $p$-value 0.23 . The mean DI value was 0.31. Out of 14 Physiology questions in CAT of Hemopoietic and immune system, $85 \%$ items were found to have $p$-value b/w $0.3-0.7$ and $15 \%$ had mean $p$-value 0.2. The mean $\mathrm{DI}$ value was 0.41 . Out of 40 Physiology questions in Semester exam, $82 \%$ items had $p$-value b/w $0.3-0.7$ and $18 \%$ items had mean $p$-value 0.18 . The mean $\mathrm{DI}$ value was 0.28 . Table 1 shows the difficulty and discrimination indices of Physiology items in respiratory system. The $p$-value of 4 items was 0.17 , $0.26,0.27$ and 0.22 respectively. The discrimination index of all 4 questions was $>0.2$. Table 1 also shows the difficulty and discrimination index of Physiology items in Hemopoietic and Immune system. The $p$-value of 2 items was 0.22 and 0.18 respectively. The discrimination index of 2 items was $>0.2$.

Table 1: Item analysis of MBBS $1^{\text {st }}$ Year Semester II CAT I respiratory system and CAT II for hemopoietic and immune system.

\begin{tabular}{|c|c|c|c|c|c|c|}
\hline $\begin{array}{l}\text { Respiratory } \\
\text { System } \\
\text { Questions }\end{array}$ & Key & Correct & Wrong & Blank & $p$-Value & $\begin{array}{l}\text { Discrimination } \\
\text { Index (DI) }\end{array}$ \\
\hline Q13 & $A$ & 17 & 83 & 0 & 0.17 & 0.31 \\
\hline Q21 & B & 26 & 74 & 0 & 0.26 & 0.26 \\
\hline Q25 & $D$ & 27 & 73 & 0 & 0.27 & 0.35 \\
\hline Q26 & C & 22 & 78 & 0 & 0.22 & 0.34 \\
\hline \multicolumn{7}{|c|}{ Hemopoietic and Immune SystemQuestions } \\
\hline Q12 & $\mathrm{D}$ & 22 & 78 & 0 & 0.22 & 0.25 \\
\hline Q21 & C & 18 & 82 & 0 & 0.18 & 0.18 \\
\hline
\end{tabular}

Table 2: Item analysis of MBBS 1st Year Semester II Respiratory and hemopoietic and immune system.

\begin{tabular}{|l|c|c|c|c|c|c|}
\hline \multicolumn{1}{|c|}{ Q. No. } & Key & Correct & Wrong & Blank & p-Value & $\begin{array}{c}\text { Discrimination } \\
\text { Index (DI) }\end{array}$ \\
\hline Q27 & C & 20 & 80 & 0 & 0.2 & 0.31 \\
\hline Q35 & A & 19 & 81 & 0 & 0.19 & 0.29 \\
\hline Q38 & E & 15 & 85 & 0 & 0.15 & 0.24 \\
\hline Q41 & B & 21 & 79 & 0 & 0.21 & 0.32 \\
\hline Q48 & C & 18 & 82 & 0 & 0.18 & 0.26 \\
\hline Q56 & D & 16 & 84 & 0 & 0.17 & 0.28 \\
\hline Q58 & B & 15 & 85 & 0 & 0.15 & 0.25 \\
\hline
\end{tabular}


Table 2 shows the difficulty and discrimination indices of Physiology items in semester II. The $p$-value of 7 items was $0.2,0.19,0.15,0.21,0.18,0.17$ and 0.15. The mean p-value was 0.18. The discrimination index of 7 items was $>0.24$. The mean DI was 0.28 .

\section{DISCUSSION}

In this study, the item analysis was done on Physiology MCQs included in CATs of courses taught in semester II MBBS as well as the semester II exam. The item analysis was matched with physiology teaching. The same faculty members were asked to develop one best Physiology MCQs on the topics they taught. In respiratory system CAT, 4 out of 15 MCQs had mean $p$-value of $<0.23$. In Hemopoietic system, 2 out of 14 MCQs had mean $p$-value of 0.2 . The topics of these $6 M C Q s$ were reviewed in relation to teaching. The quality of continuous assessment tests plays a vital role in giving feedback to teachers on their learning activities $^{13}$. With item analysis, teachers get an idea about how well a particular topic has been comprehended by the students and which topics need more time to go through ${ }^{15}$. Based on the finding of the present study, teachers customized their teaching either for content or learning strategy ${ }^{15}$. Moreover, item analysis is beneficial for increasing teachers' skills in MCQ development and indicating particular areas of the course content, which require better emphasis or clarity ${ }^{14}$. Discussing the outcome of item analysis with faculty helps in improving learning outcome ${ }^{15}$.

Optimizing student learning and academic performance is a constant challenge for medical schools $^{16}$. As assessment is an important component of student learning, assessment tools should be valid, reliable and objective and indicate various achievement levels ${ }^{12}$. Multiple-choice questions (MCQs) are a tool that is commonly used to evaluate learning performance of undergraduate students $^{15}$. In the discipline of Physiology, the concepts, terms, and structures are not readily visualized. The mechanisms need to be taught by employing distinct strategies to enhance comprehension ${ }^{17}$. Developing the skill to think critically is a fundamental aspect of undergraduate physiology education ${ }^{18}$. It is governed by appropriate use of learning and assessment strategies.

Out of 80 MCQs, there were 40 Physiology MCQs in semester II exam. Developing MCQs to assess students' knowledge thoroughly at the end of a semester is a difficult and tedioustask ${ }^{13}$. At this point, item analysis provided important diagnostic information on what undergraduate students have learned and what they have not learned ${ }^{14}$. This correlation of assessment with teaching learning was a reflection of the students' performance regarding the learning objectives taught over the specific period of time ${ }^{13}$. Item analysis and storing the MCQs with their indices allows an examiner to select MCQs of appropriate difficulty level according to the need of assessment ${ }^{16}$.

\section{CONCLUSION}

The item analysis of the majority of one best type MCQs of CATs and semester exam in Physiology had good difficulty and discrimination indices and matched with teaching and learning in Physiology.

\section{ACKNOWLEDGEMENTS}

The authors would like to acknowledge the Jinnah Medical and Dental College for facilitating the study.

\section{CONFLICT OF INTEREST}

The authors declare no conflict of interest.

\section{ETHICS APPROVAL}

The study was approved from the institutional review board of the Jinnah Medical and Dental College.

\section{PARTICIPANTS CONSENT}

Informed written consents were taken from all the participants.

\section{AUTHORS' CONTRIBUTION}

All authors contributed equally in this manuscript.

\section{REFERENCES}

1. Saint DA, Horton D, Yool A, Elliott A. A progressive assessment strategy improves student learning and perceived course quality in undergraduate physiology. Adv Physiol Educ. 2015; 39(3):218-222

2. Ferris $\mathrm{H}$, Flynn DO. Assessment in medical education; what are we trying to achieve? Int J Higher Educ. 2015; 4(2):139-144.

3. Tariq S, Tariq S, Maqsood S, Jawed S, Baig M. Evaluation of cognitive levels and item writing flaws in medical pharmacology internal assessment examinations. Pak J Med Sci. 2017; 33(4):866-870.

4. Neilson DG, Gotzsche O, Sonne O, Eika B. The relationship between immediate relevant basic science knowledge and clinical knowledge: physiology knowledge and transthoracic echocardiography image interpretation. Adv Health Sci Educ. 2012;17 (4):501-513

5. Ghosh S, Dawka V. Combination of didactic lecture with problem-based learning sessions in physiology teaching in a developing medical college in Nepal. 
Adv Physiol Educ. 2000; 24(1):8-12

6. Bhattacharya J, Chakraborty S, Thakurta TG. Learning gains through multiple choice questions in physiology. Int J Contemp Med Res. 2017; 4(1):254-260.

7. Islam ZU, Usmani A. Psychometric analysis of anatomy MCQs in modular examination. Pak J Med Sci. 2017;33 (5):1138-1143.

8. Hingorjo M, Jaleel F. Analysis of one-best MCQs: the difficulty index, discrimination index and distractor efficiency. J Pak Med Assoc. 2012; 62 (2):142-147.

9. Shahid R, Farooq Q, lqbal R. Item analysis of multiple choice questions of ophthalmology at Rawalpindi Medical University, Rawalpindi, Pakistan. Rawal Med J. 2019; 44(1):192-195.

10. Burud I, Nagandla K, Agarwal P. Impact of distractors in item analysis of multiple choice questions. Int J Res Med Sci. 2019;7(4):1136-1139.

11. Shete AN, Kausar A, Lakhkar K, Khan ST. Item analysis: An evaluation of multiple choice questions in Physiology examination. J Contemp Med Educ. 2015; 3(3):106-109.

12. Kheyami D, Jaradat A, Al-Shibani T, Ali FA. Item analysis of multiple choice questions at the department of paediatrics, Arabian Gulf University, Manama, Bahrain. Sultan Qaboos Univ Med J. 2018; 18(1):e68-e74.

13. Quaigrain K, Arhin AK. Using reliability and item analysis to evaluate a teacher-developed test in educational measurement and evaluation. Cogent Educ. 2017:4:1-11.

14. Boopathiraj C, Chellamani K. Analysis of test items on difficulty level and discrimination index in the test for research in education. Int J Soc Sci Interdiscip Res. 2013; 2(2):189-193.

15. Kolte $\vee$. item analysis of multiple choice questions in physiology examination. Int J Appl Basic Med Res. 2015;4(4):320-326.

16. Kickert R, Stegers-Jager KM, Meeuwisse M, Prinzie $P$, Arends LR. The role of the assessment policy in the relation between learning and performance. Med Educ. 2018;52(3):324-335.

17. Rehman R, Khan AN, Kamran A. Role of small group interactive sessions in two different curriculums based medical colleges. J Pak Med Assoc. 2012; 62(9):920-923.

18. Abraham RR, Upadhya S, Torke S, Ramnarayan K. Clinically oriented physiology teaching: strategy for developing critical-thinking skills in undergraduate medical students. Adv Physiol Educ. 2004; 28 (1-4):102-104. 